\title{
Orbital lesions, an exceedingly rare site of neuroendocrine tumor metastasis
}

\author{
Sara Pusceddu', Massimo Milione ${ }^{2}$, Silvia Ortolani ${ }^{3}$, Alessio Pellegrinelli² $^{2}$ Marco Brugia ${ }^{4}$, Filippo de Braud ${ }^{1}$, \\ Lorenzo Antonuzzo 4,5 \\ 'Department of Medical Oncology, Fondazione IRCCS "Istituto Nazionale dei Tumori", 20133 Milan, Italy. \\ 2Department of Phatology, Fondazione IRCCS "Istituto Nazionale dei Tumori”, 20133 Milan, Italy. \\ ${ }^{3}$ Department of Medical Oncology, Azienda Ospedaliera Universitaria Integrata, University of Verona, 37134 Verona, Italy \\ ${ }^{4}$ Department of Medical Oncology, Azienda Ospedaliera Universitaria Careggi, 50134 Firenze, Italy. \\ ${ }^{5}$ Medical Genetics, University of Siena, 53100 Siena, Italy.
}

Correspondence to: Dr. Sara Pusceddu, Department of Medical Oncology, Fondazione IRCCS “Istituto Nazionale dei Tumori”, Via G. Venezian 1,20133 Milan, Italy. E-mail: sara.pusceddu@istitutotumori.mi.it

\section{A B S T R A C T}

Neuroendocrine tumors are rare neoplasms arising primarily in the gastrointestinal tract and lung. The liver is the most common site of metastases, but these tumors can rarely metastasize to atypical sites. Surgery is the only curative approach while the optimal medical treatment is debated. From this perspective, a multidisciplinary approach for each single case becomes very important. In this report we describe the case of a male affected by a single intraorbital metastasis from a midgut well differentiated neuroendocrine tumor. The patient refused surgical removal and therefore he was at first treated with stereotactic radiotherapy and systemic treatment with a somatostatin analog (SSA). After achieving a stable disease for four months he underwent primary tumor resection. Six years after the initial diagnosis, the patient is currently stable and receiving SSA at standard dose.

Key words: Neuroendocrine tumors; orbital metastases; somatostatin analogues

\section{INTRODUCTION}

Neuroendocrine tumors are rare neoplasms derived from enterochromaffin cells, which are primarily found in the gastrointestinal tract and lung. ${ }^{[1,2]}$ Liver is the most common site of metastasis, however as survival is increasing by improved treatment options, new metastatic patterns have emerged. ${ }^{[3]}$ Ocular metastases, considered "a rarity in the rare," have now been described in neuroendocrine tumors. ${ }^{[4,5]}$ Considering the rarity of these tumors, it is clear that a multidisciplinary approach is necessary in order to obtain the best therapeutic outcome for each single patient. Here, we present a case where the integrated use of localregional and systemic treatments resulted in long-term disease stabilization, preserving the quality of life.

This case raises important issues. Considering the favorable general prognosis despite the advanced stage, treatments that maintain a good quality of life are the fundamental issues for these patients. Therefore, the alternative locoregional treatments alone (stereotactic radiotherapy) or in combination with systemic therapy, or systemic

\begin{tabular}{|l|l|}
\hline \multicolumn{2}{|c|}{ Access this article online } \\
\hline Quick Response Code: & Website: \\
\hline & www.jcmtjournal.com \\
\cline { 2 - 2 } & \\
\hline
\end{tabular}

somatostatin analog (SSA) therapy alone may constitute valid treatment options towards the goal of long-term disease stabilization and improved quality of life.

\section{CASE REPORT}

We report the case of a 65-year-old male patient, in good general conditions, with a past medical history of hypertension, diabetes mellitus and ischemic heart disease.

He presented in September 2009 complaining the recent onset of right exophthalmos.

A computed tomography (CT) scan of the head and neck documented the presence of a retroocular lesion with a maximum diameter of $28 \mathrm{~mm}$, invading both the intraconal and the extraconal space and causing optic nerve impingement. A biopsy of the lesion was performed in November 2009 through endoscopic endonasal approach and the pathologic examination diagnosed welldifferentiated neuroendocrine tumour cells.

This is an open access article distributed under the terms of the Creative Commons Attribution-NonCommercial-ShareAlike 3.0 License, which allows others to remix, tweak, and build upon the work non-commercially, as long as the author is credited and the new creations are licensed under the identical terms.

For reprints contact: service@oaepublish.com

How to cite this article: Pusceddu S, Milione M, Ortolani S, Pellegrinelli A, Brugia M, de Braud F, Antonuzzo L. Orbital lesions, an exceedingly rare site of neuroendocrine tumor metastasis. J Cancer Metasta Treat 2016;2:341-4.

Received: 20-06-2016; Accepted: 18-07-2016 

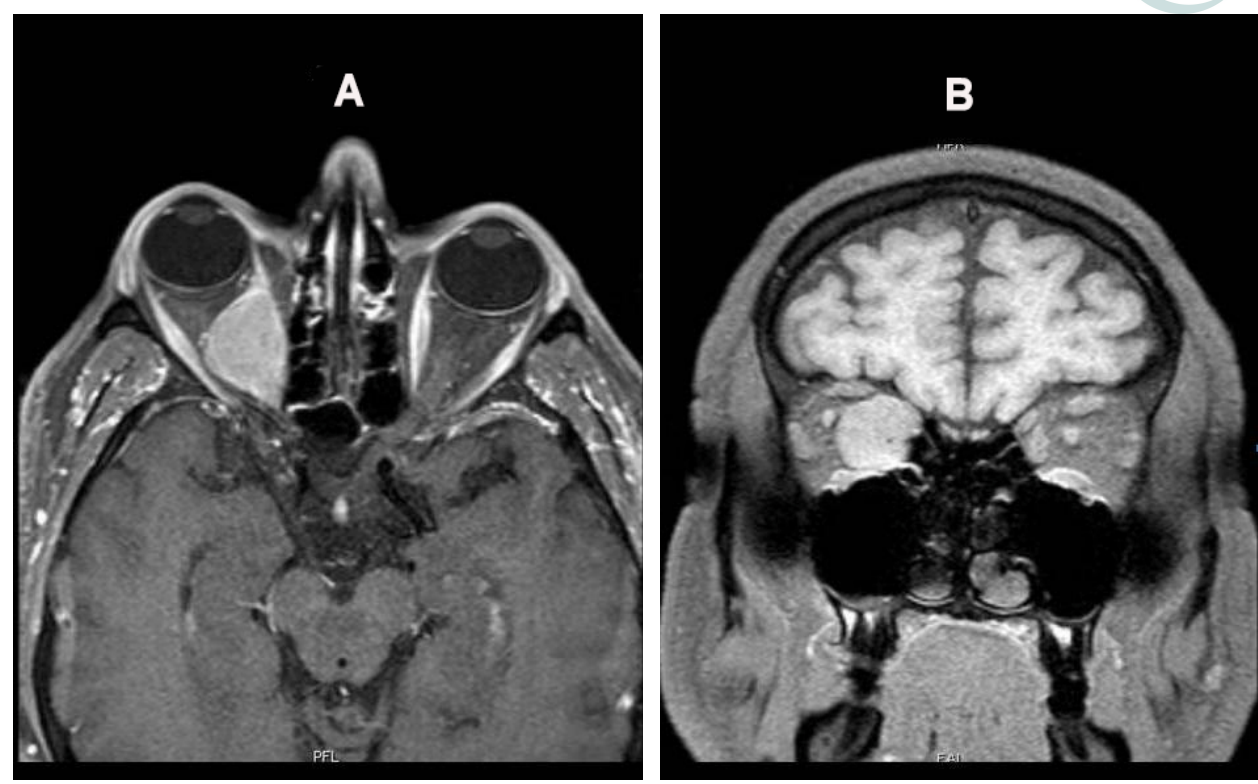

Figure 1: Intraorbital localization of well-differentiated neuroendocrine tumour (G1) of the ileo-cecal valve. MRI of the brain, head and face MRI: sagittal (A) and coronal (B) views. Lesion occupying the great part of the right orbit, and dislocating the optic nerve, though maintaining a cleavage plan from its meningeal structures. MRI: magnetic resonance imaging
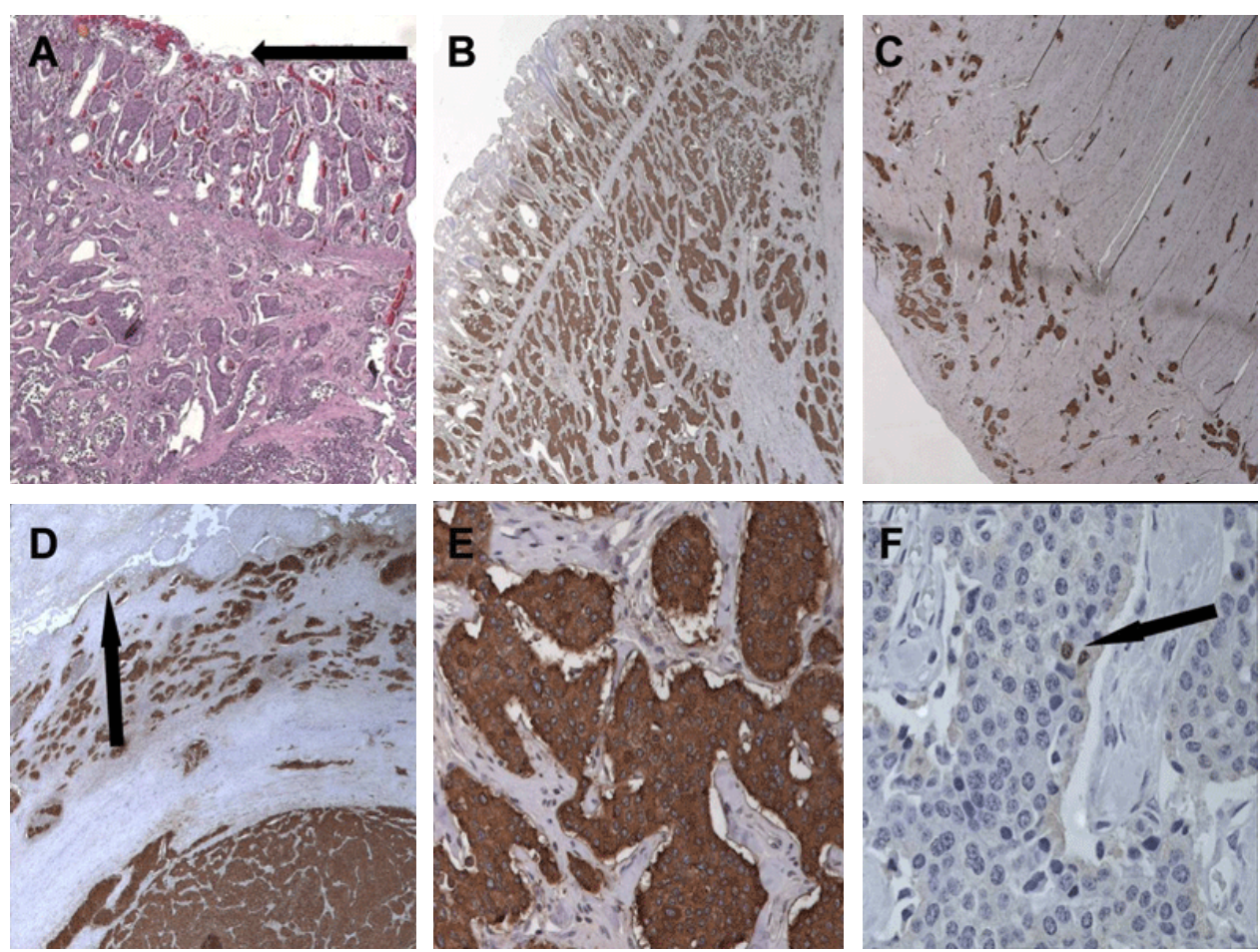

Figure 2: Well-differentiated neuroendocrine tumor (G1) of the ileo-cecal valve. (A) Mucosal ulceration (arrow, X10); (B) positivity of neoplastic nests for Chromogranin A (CgA) in the submucosa (X10); (C) muscolar layer neoplasitic invasion and positivity for $\mathrm{CgA}(\mathrm{X} 4)$; (D) piercing serosa positivity for CgA (arrow, X10); (E) serotonin stains the enterochromaffin cells (EC) (X20); (F) mindbomb homolog 1/ki-67 proliferation index below 2\% (arrow, X40)

The physical examination confirmed a slight right eyeball ptosis without significant visual function impairment; neither symptoms nor signs of carcinoid syndrome were present, the laboratory routine blood tests as complete blood count, kidney and liver function tests and electrolyte levels resulted in range.

The thorax and abdominal CT scan showed a lobulated mass with a maximum diameter of $37 \mathrm{~mm}$ at the ileocecal valve level. A magnetic resonance imaging of the brain, head and neck confirmed the presence of the previously described lesion, occupying the great part of the right orbit and dislocating the optic nerve, though maintaining a cleavage plan from its meningeal structures [Figure 1].

The Octreoscan showed pathological uptake of the tracer in the right intraorbital space and in the right iliac fossa. An endoscopic biopsy of the sub mucosal lesion found on the ileocecal valve during a pan colonoscopy confirmed the primary site of the well differentiated, neuroendocrine 

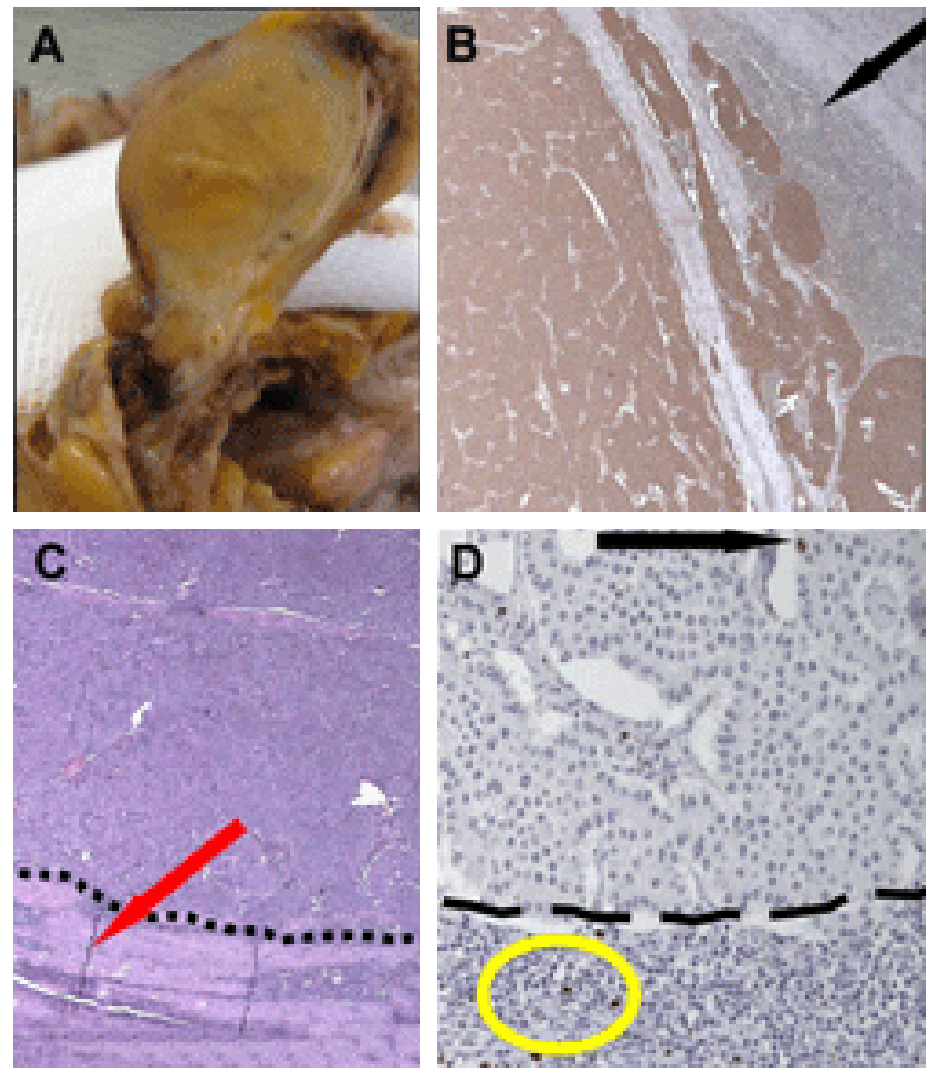

Figure 3: Lymph node metastasis from well differentiated neuroendocrine tumour (G1) of the ileo-cecal valve. (A) Metastatic mesenteric lymph node (not microscope photo); (B) evidence of nodal compression with serotonin stain (arrow, X4); (C) compression of the nodal parenchima by neoplastic cells (arrow, X4); (D) mindbomb homolog 1/ki-67 proliferation index below $2 \%$ (arrow). Non-neoplastic lymphocytes (D, oval) as internal control (X40)

tumour. Serum tumour markers such as neuron specific enolase and chromogranin $\mathrm{A}(\mathrm{CgA})$ resulted in range. The diagnostic work-up was therefore suggestive for a single, intraorbital metastasis from midgut well differentiated neuroendocrine tumour.

The patient was evaluated for radical resection of the intraorbital lesion through a transcranial excision (exenteratio orbitis), but the patient refused the orbitotomy.

He therefore underwent two 4-week courses of stereotactic radiotherapy to the right orbital metastases $(4,000 \mathrm{cGy}$ in 20 fractions) and he started a systemic treatment with Lanreotide Autogel (ATG) (fl 120 mg, 1 fl i.m. q 28 d).

The patient remained stable with persistent right exophthalmos and conjunctivitis, but without any vision deterioration after two months of radiation therapy and SSA.

He continued the SSA as maintenance treatment and after four months he underwent primary tumour resection with right hemicolectomy and lymphadenectomy. The pathology report confirmed a well-differentiated neuroendocrine tumour of ileocecal valve, G1, pT3, N1, M1 according to the ENETS/UICC TNM classification. The immunohistochemical analysis showed wide positivity for CgA, synaptophysin; serotonin and CDX2 [Figures 2 and 3].
Six years after the first diagnosis, the patient is continuing maintenance medical treatment with Lanreotide ATG 120 mg every 28 days, since stabilization of the disease.

\section{DISCUSSION}

The therapeutic strategy for neuroendocrine neoplasms (NENs) needs to be diversified according to the clinical presentation of each single case, and moreover according to its biological behaviour, due to the wide heterogeneity of these tumors.

Because of this, a multidisciplinary care team is critical for patient management starting from the earliest steps of the diagnostic workup.

Ocular metastases have been rarely described in neuroendocrine tumours; the vast majority of metastases affect the uveal tract rather than orbital space, and typically occur through haematogenous spread by carotid and ophthalmic artery. ${ }^{[4,5]}$ Data regarding survival after the diagnosis of orbital metastases of NENs are exceedingly rare. Mehta et al. ${ }^{[6]}$ describe a series of 13 patients with metastatic orbital carcinoid tumors with overall survival of $72 \%$ at 5 years and $38 \%$ at 10 years.

Considering the favorable general prognosis despite the advances stage, treatments that maintain a good quality of life with the preservation of vision are the fundamental 
issues for these patients. Therefore in advanced patients with orbital metastases, the alternative loco-regional treatments alone or in combination with systemic therapy may constitute a viable treatment alternative to a surgical excision (exenteratio orbitis). ${ }^{[7]}$

The currently proposed treatment of orbital metastases in well-differentiated NETs includes surgery, beam radiotherapy, especially for single and symptomatic lesions, peptide receptor radiotherapy or systemic medical treatment. The integration of local treatment with SSA could provide long-term disease control, preserving the patient's quality of life. Although the SSA objective response rates are limited $(5-10 \%)$, these drugs are characterized by high rates of disease stabilization, up to $50-60 \%$ in clinical trials and with optimal profiles of safety and tolerability. ${ }^{\left[{ }^{[8]}\right.}$ Moreover, the efficacy of SSA has been recently shown by two prospective, randomized, placebo-controlled trials, the PROMID and CLARINET studies. ${ }^{[9,10]}$ These studies evaluated the impact of SSA treatment (octreotide longacting release $30 \mathrm{mg}$ every 28 days and Lanreotide ATG $120 \mathrm{mg}$ every 28 days), leading to demonstration of their antiproliferative effects. The mean time to progression in the PROMID trial was 14.3 months in the octreotide LAR arm compared to 6 months in the placebo arm. ${ }^{[9]}$ In the CLARINET trial, Lanreotide ATG was associated with a significant improvement in mean progression free survival compared to placebo (progression-free survival not reached in the treatment group vs. 18 months in the placebo group) ${ }^{[10]}$ Based on these results, the use of SSA is recommended for its antiproliferative effect in well differentiated NENs with an indolent course in patients with both functioning and non-functioning tumors. SSAs represent a valid treatment option in cases where good quality of life is paramount and in which a surgical approach is not accepted, feasible or is contraindicated.

\section{Financial support and sponsorship}

Nil.

\section{Conflicts of interest}

There are no conflicts of interest.

\section{Patient consent}

Obtained.

\section{Ethics approval}

The patient was treated within the standards of our institute and the report was approved.

\section{REFERENCES}

1. Modlin IM, Lye KD, Kidd M. A 5-decade analysis of 13,715 carcinoid tumors. Cancer 2003;97:934-59.

2. Pinchot SN, Holen K, Sippel RS, Shen H. Carcinoid tumors. Oncologist 2008; 13:1255-69.

3. Zuetenhorst JM, Taal BG. Metastatic carcinoid tumors: a clinical review. Oncologist 2005;10:123-31.

4. Karcioglu ZA. Orbital tumours: Diagnosis and treatment. 2nd ed. New York: Springer; 2005. p. 187-9.

5. Peixoto RD, Lim HJ, Cheung WY. Neuroendocrine tumor metastatic to the orbit treated with radiotherapy. World J Gastrointest Oncol 2013;5:177-80

6. Mehta JS, Abou-Rayyah Y, Rose GE. Orbital carcinoid metastases. Ophthalmology 2006;113:466-72.

7. Borota OC, Kloster R, Lindal S. Carcinoid tumour metastatic to the orbit with infiltration to the extraocular orbital muscle. APMIS 2005;113:135-9.

8. Modlin IM, Pavel M, Kidd M, Gustafsson BI. Review article: somatostatin analogues in the treatment of gastroenteropancreatic neuroendocrine (carcinoid) tumours. Aliment Pharmacol Ther 2010;31:169-88.

9. Rinke A, Müller HH, Schade-brittinger C, Klose KJ, Barth P, Wied M, Mayer C, Aminossadati B, Pape UF, Bläker M, Harder J, Arnold C, Gress T, Arnold R; PROMID Study Group. Placebocontrolled, double-blind, prospective, randomized study on the effect of octreotide LAR in the control of tumor growth in patients with metastatic neuroendocrine midgut tumors. $J$ Clin Oncol 2009;27:4656-63.

10. Caplin ME, Pavel M, Ćwikła JB, Phan AT, Raderer M, Seedláčková E, Cadiot G, Wolin EM, Capdevila J, Wall L, Rindi G, Lanley A, Martinez S, Blumberg J, Ruszniewski P. Lanreotide in Metastatic Enteropancreatic Neuroendocrine Tumors. $N$ Engl J Med 2014;371:224-33. 